\title{
Artificial neural network inspired by quantum computing solutions using the movement model of the PR-02 robot
}

\author{
Jerzy Tchórzewski ${ }^{1, *}$, Dariusz Ruciński ${ }^{1}$, and Przemysław Domański ${ }^{1}$ \\ ${ }^{1}$ Institute of Computer Sciences, University of Natural Sciences and Humanities, 3-Maja 54, 08-110 Siedlce, Poland
}

\begin{abstract}
The paper proposes a new method of quantum computing using control and systems theory as well as matrix-quantum computing. The algorithm developed on the basis of the PR-02 robot's arm's movement was implemented in using the Neural Network Toolbox. The application of the neural model instead of the analytic model allowed for obtaining the improvement of the trajectory of the PR-02 robot's arm movement, while the application of the quantum artificial neural network for the assumed number of quasi-parallel computations equal 1000 did not result in the improvement of the model.
\end{abstract}

\section{Introduction}

Currently, more and more accurate modeling methods are being sought, especially smart grid systems. High accuracy is achieved using neural and evolutionary modeling methods. Obtained, among others detailed neural models of the Polish Electric Power Exchange [12] and models of the PR-02 robot movement [3]. It is possible to increase the accuracy of models when inspiring artificial intelligence methods with quantum computing solutions, which is associated with the slowing down of computations due to quasi parallel processing [2-3]. In this spirit, the example of the movement of the end of the robot's arm PR-02 shows that the quantum-inspired artificial neural network (ANN) increases the accuracy of the end position of the robot arm at the starting point and at the end point and raises hope for a more precise trajectory.

\section{Quantum computing}

Fundamentals of the control and systems theory were used to describe the quantum-inspired artificial neural network [4], in particular, the definition of the state of the system, which was used as the basis for determination of mixed states of a quantum number. Using numerical data related to the changes of the four above mentioned parameters, described in detail in works, i.a. [2-3] the artificial neural network was designed and taught the movement of the PR-02 movement, followed by the development of the method of quantization, dequantization and quantum computing, thus making an attempt to improve the accuracy of the neural model. Quantum computing was interpreted based on the example of a single neuron, described by, e.g. the activation function of the sigmoidal tangent type of the i-th neuron of k-th weights layer of the perceptron artificial neural network [2-3], as follows:

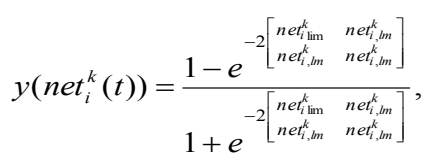

where $n e t_{i}^{k}-$ quantum summator of the i-th neuron in the $\mathrm{k}$-th weights layer defined as the sum of weighted quantum values of input signals to the k-th neuron layer, $n e t_{i, m}^{k}$ - the element of the summator net ${ }_{i}^{\mathrm{k}}$ with the index $1 \mathrm{~m}$ as quantum weighted input signal to the k-th neuron layer of ANN with the clean state nature resulting from two mixed states.

\section{Analytical model of the PR-02 robot}

Robots of this type feature uncomplicated trajectory programming and a simple power-supply system [6]. Simple equation of the PR-02 robot's grab kinematics movement is presented using a transformation graph using the following form of matrix $\mathrm{X}$ :

$$
\mathbf{X}=\left[\begin{array}{cccc}
C_{1} S_{4} & C_{1} C_{4} & -S_{1} & l_{2} C_{1}-S_{1} \lambda_{3} \\
S_{1} S_{4} & S_{1} C_{4} & C_{1} & l_{2} C_{1}+S_{1} \lambda_{3} \\
C_{4} & -S_{4} & 0 & l_{3}+\lambda_{5} \\
0 & 0 & 0 & 1
\end{array}\right]
$$

with values of cosines and sines of respective angles at the joint being denoted as the values of parameters $C_{i}$ and $\mathrm{S}_{\mathrm{i}}$, and described in detail in table $1[2-3,5]$. Change of H-D parameters directly controls the movement of the PR-02 robot's arm's endpoint; as a result of which, the robot's arm's end draws in the Cartesian space XYZ the trajectory shown in Table $2[2-3,5]$.

Table 1. Values of Hartenberg-Denevit (H-D) parameters used to describe the PR-02 manipulator [5].

\begin{tabular}{|c|c|c|c|c|}
\hline No & $\alpha_{\mathrm{i}}\left[^{\circ}\right]$ & $l_{\mathrm{i}}[\mathrm{m}]$ & $\lambda_{\mathrm{i}}[\mathrm{m}]$ & $\theta_{\mathrm{i}}\left[{ }^{\circ}\right]$ \\
\hline 1 & 0 & 0 & 0 & $-300 \div 0$ \\
\hline 2 & -90 & -0.11 & $0 \div 0.200$ & 0 \\
\hline 3 & 0 & 0 & $0.367 \div 0.676$ & -90 \\
\hline 4 & 0 & 0 & 0 & $0 \div 360$ \\
\hline
\end{tabular}




\section{Neural and quantum PR-02 models}

In order to obtain the neural model of the PR-02 robot's arm's end movement, a perceptron artificial neural network was designed-described in detail in works e.g. [1-3,6-7]. Values of parameters presented in table 1 were used for the purpose of training the Artificial Neural Network (ANN), with the number of epochs equal 1164. As a result of training ANN, MSE error of the order of magnitude $10^{-3}-10^{-4}$ was obtained after approx. 80 epochs (train) [3]. A model of quantum artificial neural network is presented in fig. 1, and its subsystems in works [5-7].

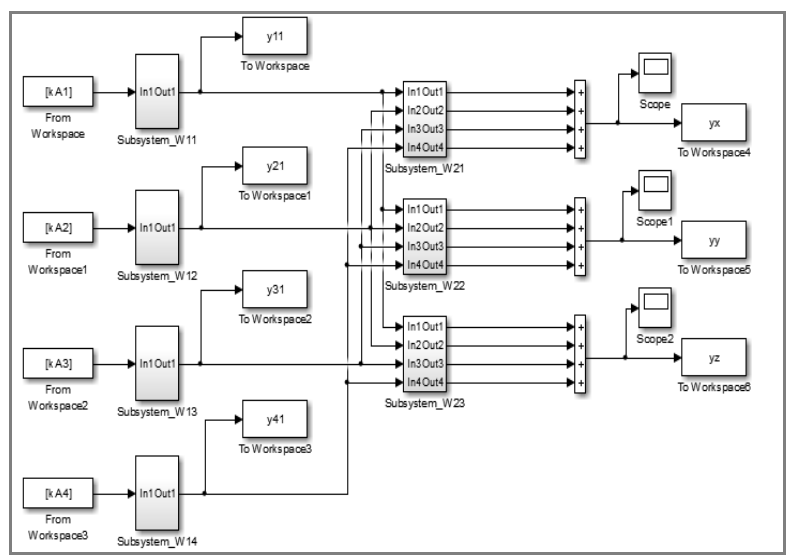

Fig. 1. A model of the Quantum ANN [3].

\section{The behavior of the PR-02 models}

The comparison of functioning of the three models of the PR-02 robot's arm's end movement, i.e. the analytic model, the neural model and the quantum model (neural, quantum computer science-inspired model - table 2), showed that the neural model obtained as a result of training the ANN the movement of the robot's arm's end was very similar to the analytic model. As a result of the calculations, the corresponding coordinates of the end point of the robot arm's trajectory for calculations were obtained: analytical - Pr $(0,20001,0,7,074,20001)$, neural
- Pn $(0,277556,0,92218,0,189762)$ and quantum - pq $(0.112611,0.867,0.200009)$.

Noteworthy are the better results regarding the position of the end point trajectory of the PR- 02 robot arm received for quantum calculations than for the neuronal calculations. The Euclidean distance determined in relation to the real point is smaller for the Quantum Artificial Neural Network and amounts to 0.087398624, while for the Artificial Neuron Network - 0.090151236. Unfortunately, it turned out that for 1000 quasi parallel calculations, the course of the trajectory of the neural model inspired by quantum computation was similar but not continuous. It was caused by a small number of quasi-parallel calculations considering the quantum computer science requirements (occurrences of mixed states with infinite number), hence, it would be necessary to increase the number of mixed states both in quantization and in quantum computing in further research, at least by one order of magnitude, e.g. to 10 or even 100 thousand of mixed quantum states and quantum calculations. The research is being continued.

\section{References}

1. D. Ruciński, Studia Informatica. Systems and Information Technology 1-2 (21), UPH, 63-83 (2017)

2. J. Tchórzewski, D. Ruciński, Pozn. Univ. Technol. Acad. J. Electr. Eng 96, 21-32 (2018)

3. J. Tchórzewski, P. Domański, Pozn. Univ. Technol. Acad. J. Electr. Eng 96, 33-46 (2018)

4. T. Kaczorek, Basics of Control Theory, (WNT, Warszawa, 2005)

5. T. Szkodny, Kinematics of industrial robots, (Publisher of the SUT, Gliwice, 2013)

6. S. Osowski, Neural networks for information processing, (OW PW, Warszawa, 2013)

7. R. Tadeusiewicz, M. Szaleniec, Lexicon of neural networks, Publisher of Foundation "Project Science Leksykon", (Wrocław, 2015)

Table 2. Trajectories of the PR-02 robot's arm: analytic (a), neural (b), quantum-neural (c) models. Source: [3,5-7].

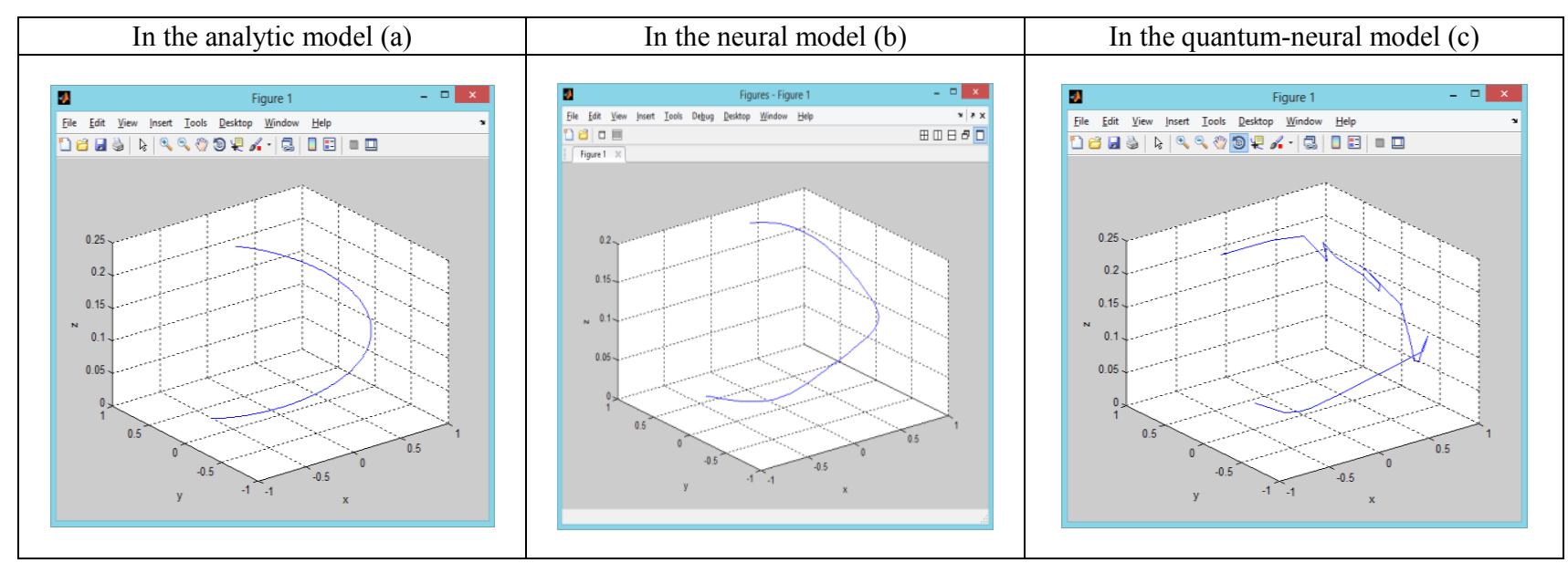

